\title{
A piecewise affine PI controller for buck converter generated DC motor
}

\author{
Mohd Ikhmil Fadzrizan Mohd Hanif, Mohd Helmi Suid, Mohd Ashraf Ahmad \\ Faculty of Electrical and Electronics Engineering, Universiti Malaysia Pahang, Malaysia
}

\begin{tabular}{l}
\hline Article Info \\
\hline Article history: \\
Received Sep 29, 2018 \\
Revised Jan 16, 2019 \\
Accepted Mar13, 2019 \\
\hline
\end{tabular}

Keywords:

Piecewise Affine ProportionalIntegral

Buck converter DC motor

Safe Experimentation

Dynamics (SED)

\begin{abstract}
This paper presents a new Piecewise Affine Proportional-Integral (PA-PI) controller for angular velocity tracking of a buck converter generated DC motor. A Safe Experimentation Dynamics (SED) algorithm is employed as a data-driven optimization tool to find the optimal PA-PI controller parameters such that the integral square of error and input are reduced. The essential feature of the PA-PI controller is that the parameters of proportional and integral gains are adaptive to the error variations according to the Piecewise Affine (PA) function. Moreover, the proposed PA function is expected to provide better control accuracy than the other existing variable structure PID controller. In order to verify the effectiveness of the PA-PI controller, a widely known buck converter generated DC motor is considered. The performances of the proposed controller are observed in terms of the integral square of error and input, and the responses of the angular velocity and duty ration input. The simulation results verify that the proposed PA-PI controller yields higher control accuracy than the other existing controllers of buck converter generated DC motor.
\end{abstract}

Copyright $(0) 2019$ Institute of Advanced Engineering and Science. All rights reserved.

\section{Corresponding Author:}

Mohd Ashraf Ahmad,

Faculty of Electrical and Electronics Engineering,

University Malaysia Pahang,

Pekan, 26600, Pahang, Malaysia.

Email: mashraf@ump.edu.my

\section{INTRODUCTION}

Recently, various applications, such as robotic manipulator, drones, conveyor belts, and electric cranes, require a high precision DC motors. Meanwhile, there are large numbers of standard pulse width modulation (PWM) applications for DC motor execution. Nevertheless, this technique degrades the precision motion of the DC motor owing to intractable switching strategy that resulting to jerk response in the voltage and current of the DC motor [1]. As a solution to the problem, a DC-DC buck converter is adopted to provide a smooth tracking motion of the DC motor. In particular, it can track the required trajectory of both angular velocity and position trajectory through regulating an input voltage only. In other words, the selection of good control scheme is very important to provide a high precision of motion of the DC motor fed by the DCDC buck converter.

To the best of our knowledge, a variety of approaches have been proposed in regulating buckconverter generated DC motor. Firstly, a feedback controller with energy shaping and damping injection [2] is used to control a $4^{\text {th }}$ order model of buck converter generated DC motor. Next, a flatness based method is adopted for angular velocity tracking of the DC motor fed by the DC-DC buck converter in [1], [3] and [4]. Here, the controller design in [4] is derived from the simplified $2^{\text {nd }}$ order model, while the controller in [1] and [3] are derived from the $4^{\text {th }}$ order model. Moreover, a sliding mode controller with delta modulation based GPI is reported in [5]. In particular, the simplified mathematical model in [4] is applied. Furthermore, a backstepping controller with non-adaptive and adaptive mechanisms is designed based on $4^{\text {th }}$ order model 
[6]. Their work claims that the adaptive mechanism is significant in improving the control performance even with load torque variations. Other control strategies of DC motor fed by DC-DC buck converter are LQR and PI controllers [7], two-stage control with differential flatness [8], Ho controller [9], robust control law with active disturbance rejection [10], neural network controller [11], and hybrid PI with Fuzzy controller [12].

Based on the stated control schemes, most of the controller techniques have employed the modelbased control schemes, which are synthesized based on the $2^{\text {nd }}$ or $4^{\text {th }}$ order model. Nevertheless, it is hard to employ the controller derived from the model in real experiment of buck-converter generated DC motor plant owing to some justifications. These are the unmodeled dynamic problems, inaccuracy of the simplified model, and massive gap between real applications and control theory. Thence, a data-driven controller, which is derived from the output and input data of the plant in the absence of any knowledge of the plant, may become a promising idea. Meanwhile, the data-driven PID control scheme is mostly preferred and established controller structure owing to its simplicity in design and easy to be implemented [13]. Moreover, the control accuracy of the standard PID control scheme can be enhanced by upgrading it to nonlinear PID [14]-[19]. Therefore, it is significant to assess the effectiveness of the data-driven nonlinear PID controller for input tracking of the angular velocity of DC motor fed by the DC-DC buck-converter.

The paper presents the data-based Piece-wise Affine PI controller for input tracking of angular velocity of DC motor fed by the DC-DC buck converter. Specifically, the Piecewise Affine PI, which is in the family of variable structure PID, is expected to provide better control accuracy than the sigmoid based PID in [19]. This is because our proposed variable structure, which is in a piece-wise affine form, is more general and not restrict to the sigmoid function. Thence, it proffers high possibility to enhance the control accuracy by using the time-varying PI gains according to the changes of angular velocity error with respect to the optimal Piecewise Affine function. Moreover, the study in [19] does not includes an explicit description of data-driven controller tuning, while in this study, a complete data-driven control synthesize is shown. Specifically, a safe experimentation dynamics (SED) algorithm [20] is employed to obtain the Piecewise Affine PI gains that minimize the angular velocity error. The SED algorithm is widely established to be a significant optimization tool owing to its capability in solving variety of optimization problems with fast convergence speed even for large number of parameters tuning [21]-[25]. Next, the proposed data-based Piecewise Affine PI controller design is verified to a widely established model of buck-converter generated DC motor [1]. The control performances of the Piece-wise Affine PI controller based SED are analyzed in terms of duty cycle input energy and angular velocity trajectory tracking. Lastly, a performance comparison between the Piecewise Affine PI controller based SED and the conventional PI [7] and the sigmoid based PI [19] is presented.

\section{BUCK CONVERTER GENERATED DC MOTOR}

The complete $4^{\text {th }}$ order mathematical model of buck converter generated DC motor, which is taken from [1], is given by

$$
H=\left[\begin{array}{l|l}
A & B \\
\hline C & D
\end{array}\right]
$$

where

$$
\begin{gathered}
A=\left[\begin{array}{cccc}
-\frac{R_{L}}{L} & -\frac{1}{L} & 0 & 0 \\
\frac{1}{C} & 0 & -\frac{1}{C} & 0 \\
0 & \frac{1}{L_{M}} & -\frac{R_{M}}{L_{M}} & -\frac{K_{E}}{L_{M}}
\end{array}\right], B=\left[\begin{array}{c}
\frac{U_{e}}{L} \\
0 \\
0 \\
0
\end{array}\right], \\
C=\left[\begin{array}{cccc}
0 & 0 & 0 & 1
\end{array}\right],
\end{gathered}
$$

The detailed of the variables of the plant in (1) is given in Table I. Note that the input $u(t)$ of the plant is a duty ratio $\delta \in[0,1]$, while the angular velocity $\omega$ is the output of the plant $y(t)$.

Int J Pow Elec \& Dri Syst, Vol. 10, No. 3, Sep 2019 : $1419-1426$ 
Table 1. Variables of buck converter generated DC motor

\begin{tabular}{llll}
\hline Variables & Symbol & Value & Unit \\
\hline Inductor of DC motor & $L_{M}$ & $8.9 \times 10^{-3}$ & $\mathrm{H}$ \\
Resistor of DC motor & $R_{M}$ & 6 & $\mathrm{Ohm}$ \\
Electromagnetic source & $K_{E}$ & 0.0517 & $\mathrm{Vs} / \mathrm{rad}$ \\
Tachogenerator gain & $K_{M}$ & 0.0517 & $\mathrm{Nm} / \mathrm{A}$ \\
Moment of inertia & $J_{M}$ & $7.95 \times 10^{-6}$ & $\mathrm{Kg}-\mathrm{m}^{2}$ \\
Input voltage & $U_{e}$ & 24 & $\mathrm{~V}$ \\
Inductor & $L$ & $1.33 \times 10^{-6}$ & $\mathrm{H}$ \\
Resistor of coil windings & $R_{L}$ & 0.2 & $\mathrm{Ohm}$ \\
Capacitor & $C$ & $470 \times 10^{-6}$ & $\mathrm{~F}$ \\
\hline
\end{tabular}

\section{PROBLEM FORMULATION}

Figure 1 shows the Piecewise Affine PI controller (PA-PI) for buck converter generated DC motor. Here, the symbols $r(t), u(t), y(t)$ and $e(t)$ are the desired angular velocity trajectory, the control input, the actual angular velocity response, and the error between desire and actual angular velocity, respectively. The plant $H$ is defined as the buck converter generated DC motor system as presented in the previous section. The PA-PI controller is expressed by

$$
C(s)=P(t)+\frac{I(t)}{s}
$$

where

$$
P(t)=\left\{\begin{array}{ccc}
c_{0}^{P}+M_{1}^{P}\left(e(t)-w_{0}^{P}\right) & \text { if } \quad w_{0}^{P} \leq e(t) \leq w_{1}^{P}, \\
c_{1}^{P}+M_{2}^{P}\left(e(t)-w_{1}^{P}\right) & \text { if } \quad w_{1}^{P} \leq e(t) \leq w_{2}^{P}, \\
& \vdots & \\
c_{l-1}^{P}+M_{l}^{P}\left(e(t)-w_{l-1}^{P}\right) & \text { if } \quad w_{l-1}^{P} \leq e(t) \leq w_{l}^{P},
\end{array}\right.
$$

where $M_{k}^{P}=\left(c_{k}^{P}-c_{k-1}^{P}\right) /\left(w_{k}^{P}-w_{k-1}^{P}\right)(k=1,2, \ldots, l)$ are the segment slopes, $w_{k}^{P}(k=0,1, \ldots, l) \in \mathrm{R}$ are the given input points for the gain $P(t)$ satisfying $w_{0}^{P}<w_{1}^{P}<\cdots<w_{l}^{P}$ and connecting the line segments, and $c_{k}^{P}(k=0,1, \ldots, l)$ are the output points corresponding to each input point. For simple notation, let define $\boldsymbol{w}^{P}=\left[\begin{array}{llll}w_{0}^{P} & w_{1}^{P} & \cdots & w_{l}^{P}\end{array}\right]^{\mathrm{T}}$. The illustration of the piecewise affine functions for the gain $P(t)$ is shown in Figure 2. In this study, it is assumed that $P(t)$ is one-to-one map to $e(t)$ and $P(t)=0$ if $e(t)=0$. Thence, $c_{0}^{P}=0$ for $w_{0}^{P}=0$. Note that a similar piece-wise affine function is applied to integral gain $I(t)$. Next, the control performance assessment for the control system block diagram in Figure 1 is given by

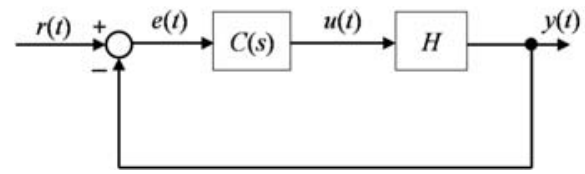

Figure 1. Control system block diagram of buck converter generated DC motor 


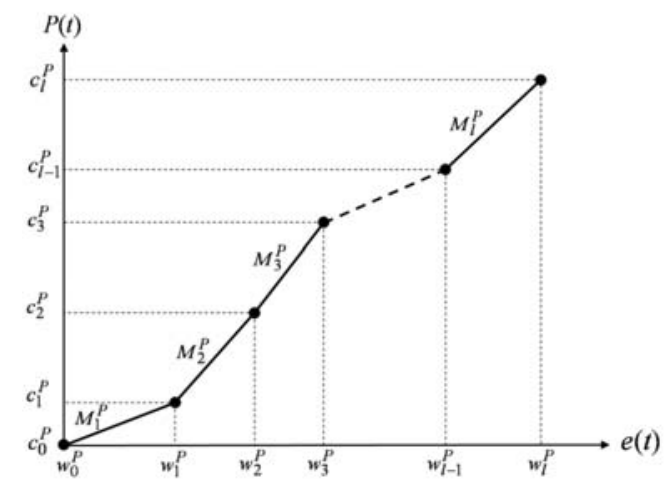

Figure 2. Piecewise affine function for the gain $P(t)$

$$
Q(P(t), I(t))=\omega_{1} \int_{t_{0}}^{t_{f}} e(t)^{2} d t+\omega_{2} \int_{t_{0}}^{t_{f}} u(t)^{2} d t
$$

Where $t_{0} \in\{0\} \cup \mathrm{R}_{+}$, and $t_{f} \in \mathrm{R}_{+}$. Note that the time interval $\left[t_{0}, t_{\mathrm{f}}\right]$ corresponds to the period for the performance evaluation. The symbols $\omega_{1}$ and $\omega_{2}$ correspond to weighting coefficients, which are given by the designer. The first term in (3) corresponds to the tracking error, while the second means the control input energy. Here, the values of $\omega_{1}$ and $\omega_{2}$ are selected in a similar way to the standard Linear Quadratic Regulator (LQR) problem. Finally, the problem statement is described by:

Problem 3.1. Consider the control system block diagram in Figure 1. Then, find the PA-PI controller $C(s)$ in (2) such that the control performance assessment $Q(P(t), I(t))$ is minimized based on the $u(t)$ and $y(t)$ data.

Remark 3.1. The proposed PA-PI controller is expected to produce better control performance from our previous version of sigmoid based PI in [19]. This is because the nonlinear PI gains in the proposed PA-PI are not restricted to only sigmoid-based function. Though it looks that we can handle general class of nonlinear subsystems PI gains by adopting so many basis functions (such as piece-wise affine functions).

\section{PA-PI CONTROLLER BASED SAFE EXPERIMENTATION DYNAMICS}

In this section, the main solution of the Problem 3.1 is explained. Firstly, an overview of the Safe Experimentation Dynamics (SED) algorithm is presented. Secondly, it is shown on how to apply the SED for tuning the parameters of PA-PI such that the control performances index in (4) is reduced.

\subsection{Overview of safe experimentation dynamics}

Safe Experimentation Dynamics (SED) is a game theoretic method that defines each element of the design variable as a player [20]. Here, the random motion of each player is decided based on pre-determined probability such that an optimal goal or design variable is achieved, which corresponds to the minimum value of the loss function. In particular, let the optimization problem is given by

$$
\min _{\boldsymbol{x} \in \mathrm{R}^{n}} L(\boldsymbol{x})
$$

where $x \in \mathrm{R}^{n}$ is the design variable, and $L: R^{n} \rightarrow R$ is denoted by the loss function. The SED algorithm iteratively updates $x \in \mathrm{R}^{n}$ using the updated law

$$
x_{i}(\tau+1)=\left\{\begin{array}{cc}
f\left(\bar{x}_{i}-\lambda r_{2}\right) & \text { if } r_{1} \leq E, \\
\bar{x}_{i} & \text { if } r_{1}>E,
\end{array}\right.
$$

for $\tau=0,1, \ldots$ where $r_{1}$ and $r_{2}$ are independent random numbers which uniformly distributed between the ranges of $[0,1], E$ is a scalar that defines the probability of using the new updated design variable, $\lambda$ 
represents the step size gain of the design variable, $x_{i}$ is the $i$-th element of $x \in R^{n}, \bar{x}_{i}$ is the $i$-th element of $\bar{x} \in$ $R^{n}$, and $\bar{x} \in R^{n}$,is denoted as the current best design variable vector. In (6), the function $f$ is given by

$$
f(\cdot)=\left\{\begin{array}{ccc}
x_{\mathrm{up}} & \text { if } & \bar{x}_{i}-\lambda r_{2}>x_{\mathrm{up}}, \\
\bar{x}_{i}-\lambda r_{2} & \text { if } & x_{\mathrm{lo}} \leq \bar{x}_{i}-\lambda r_{2} \leq x_{\mathrm{up}}, \\
x_{\mathrm{lo}} & \text { if } & \bar{x}_{i}-\lambda r_{2}<x_{\mathrm{lo}},
\end{array}\right.
$$

where $x_{u p}$ and $x_{10}$ are pre-defines upper and lower bound values of design variable, respectively. The important feature of SED is that it can provide a stable convergence due to its capability to keep the best design variable during the tuning process. Moreover, the SED uses a fixed interval step size, which is independent of the number of iterations. Therefore, it will be useful data-driven optimization tool for the case when disturbances or uncertainties or stochastic delay occur during the tuning process. In this study, the proposed SED algorithm terminates using pre-specified stopping criterion $\tau_{\max }$ with the solution $\boldsymbol{x}^{*}=\arg \min _{\boldsymbol{x} \in\{\boldsymbol{x}(0), \boldsymbol{x}(1), \ldots, \boldsymbol{x}(\tau+1)\}} L(\boldsymbol{x})$.

\subsection{Application of SED to tune PA-PI controller}

In this section, it is described on how to employ the SED algorithm in Section 4.1 for tuning the proposed PA-PI controller of buck converter generated DC motor. Initially, the design variable with respect to the PA-PI controller gains is defined by

$$
\boldsymbol{v}=\left[c_{1}^{P} \cdots c_{l}^{P} c_{1}^{I} \cdots c_{l}^{I}\right]^{\mathrm{T}}
$$

where $v \in \mathrm{R}^{\rho}$ for $\rho=2 l$. Next, a logarithmic function is utilized for every element of $x \in \mathrm{R}^{\rho}$ to provide a fast design variable searching. In particular, the new setting of the design variable is $\left[\begin{array}{llll}v_{1} & v_{2} & \cdots & v_{\rho}\end{array}\right]^{\mathrm{T}}=\left[\begin{array}{lllll}10^{x_{1}} & 10^{x_{2}} & \cdots & 10^{x_{\rho}}\end{array}\right]^{\mathrm{T}}$ with the loss function $L\left(\left[\begin{array}{llll}10^{x_{1}} & 10^{x_{2}} & \cdots & 10^{x_{\rho}}\end{array}\right]^{\mathrm{T}}\right)$. Lastly, the stepby-step procedure to apply the SED algorithm to PA-PI controller is stated as follow:

Step 1: Set the initial design variable $\boldsymbol{x}(0)$ with pre-specified $\tau_{\max }$ and $x_{i}=\log v_{i}$ for $i=1,2, \ldots, \rho$. Select

Step 2: Execute the SED algorithm in (6) by setting the design variable with $v$ in (8) and $L:=Q$.

Step 3: After $\tau_{\max }$ iterations, obtain the optimal design variable $x^{*} \in \mathrm{R}^{\rho}$ and apply to $C(s)$ in (2).

\section{NUMERICAL RESULTS}

In this section, the effectiveness of the proposed PA-PI controller based SED is presented. The model of the buck-converter generated DC motor in (9) is used. The desired angular velocity used in this numerical example is based on the tangent hyperbolic function from [19]. The design variable of Piecewise Affine PI controller $C(\mathrm{~s})$ is given in the second column of Table II with $\boldsymbol{w}^{P}=\boldsymbol{w}^{I}=\left[\begin{array}{llllll}0 & 3 & 6 & 9 & 12 & 15\end{array}\right]^{\mathrm{T}}$

and $c_{0}^{P}=c_{0}^{I}=0$. Our aim is to obtain the optimal $v \in \mathrm{R}^{\rho}$ that minimizes the loss function $Q$ in (3) for $\omega_{1}=10, \omega_{2}=1, t_{0}=0, t_{f}=0.25$ and $\tau_{\max }=1000$. The coefficients of the SED are set as $E=0.7$ and $\lambda=$ 0.01 with $x_{\text {up }}=3$ and $x_{\mathrm{lo}}=-3$. Note that the initial design variable in Table II is selected based on the values of conventional PI controller in [7]. For example, the value of the proportional gain from [7], which is 0.0069 , is multiplied with $\boldsymbol{w}^{P}$ to obtain the first five values of vector $\boldsymbol{v}(0)$ in the column 4 of Table II.

The optimal design variable $\boldsymbol{v}^{*} \in \mathrm{R}^{\rho}$ is tabulated in Table 2 . Based on the obtained $\boldsymbol{v}^{*} \in \mathrm{R}^{\rho}$, the resulting Piecewise Affine function for the proportional and integral gains can be observed in Figures 3 and 4 , respectively. Note that, the proportional gain requires high magnitude of gain for the angular velocity error ranges $0-3$ and $13-15$. Meanwhile, the integral gain requires high magnitude of gain for the angular velocity error between 0 and 3 . It shows that by employing time varying $P(t)$ and $I(t)$ gains according to the changes of error, it will offer more rooms for the controller improvement. Moreover, Figures 5 and 6 show the responses of the angular velocity and the duty ratio for the PA-PI controller in comparison the conventional PI and Sigmoid-PI controllers. Note that, the reference is represented by the dot black line, the PI controller is represented by thick grey line, the sigmoid-based PI controller is represented by the dash black line and the PA-PI controller is represented by the thick black line. In general, the responses clarify that the proposed PA-PI based SED yields better angular velocity trajectory tracking with slightly minimum duty ratio than other existing methods. Specifically, from the magnified picture, the actual response of $y(t)$ is much closer to the reference $r(t)$ than other controllers. Similarly, the response of duty ratio of the PA-PI controller 
is slightly lower than the recent sigmoid based PI controller. This is supported by the numerical analysis of the integral square of error and input as tabulated in Table 3. It is shown that the values of the integral square of error and input for PA-PI controller are much lower than other controllers.

Table 2. PA-PI design variables

\begin{tabular}{lccccc}
\hline $\boldsymbol{v}$ & PA-PI Parameters & $x(0)$ & $\boldsymbol{v}(0)=10^{\mathbf{x}(0)}$ & $x^{*}$ & $\boldsymbol{v}^{*}=10^{\mathbf{x}^{*}}$ \\
\hline$v_{1}$ & $c_{1}^{P}$ & -1.6840 & 0.0207 & -1.0187 & 0.0958 \\
$v_{2}$ & $c_{2}^{P}$ & -1.3830 & 0.0414 & -1.3467 & 0.0450 \\
$v_{3}$ & $c_{3}^{P}$ & -1.2069 & 0.0621 & -1.3887 & 0.0409 \\
$v_{4}$ & $c_{4}^{P}$ & -1.0820 & 0.0828 & -1.3123 & 0.0487 \\
$v_{5}$ & $c_{5}^{P}$ & -0.9851 & 0.1035 & -0.9742 & 0.1061 \\
$v_{6}$ & $c_{1}^{I}$ & 0.0757 & 1.1904 & 1.5320 & 34.0338 \\
$v_{7}$ & $c_{2}^{I}$ & 0.3767 & 2.3808 & 1.2557 & 18.0183 \\
$v_{8}$ & $c_{3}^{I}$ & 0.5528 & 3.5712 & 0.8146 & 6.5247 \\
$v_{9}$ & $c_{4}^{I}$ & 0.6778 & 4.7616 & 0.8735 & 7.4739 \\
$v_{10}$ & $c_{5}^{I}$ & 0.7747 & 5.5920 & 0.3712 & 2.3505 \\
\hline
\end{tabular}

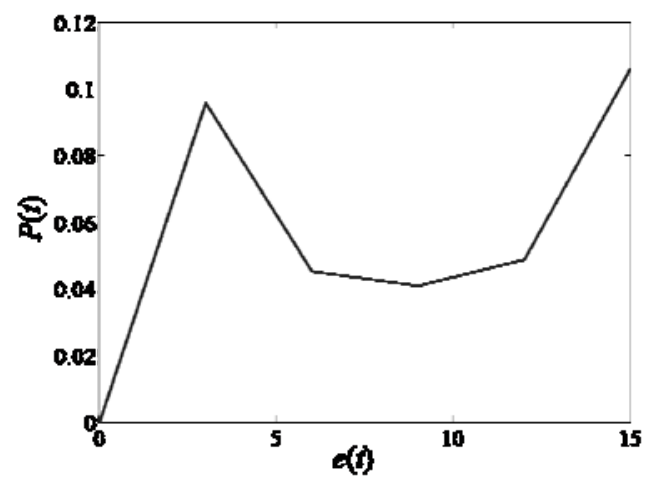

Figure 3. The resulting Piecewise Affine function for the gain $P(t)$

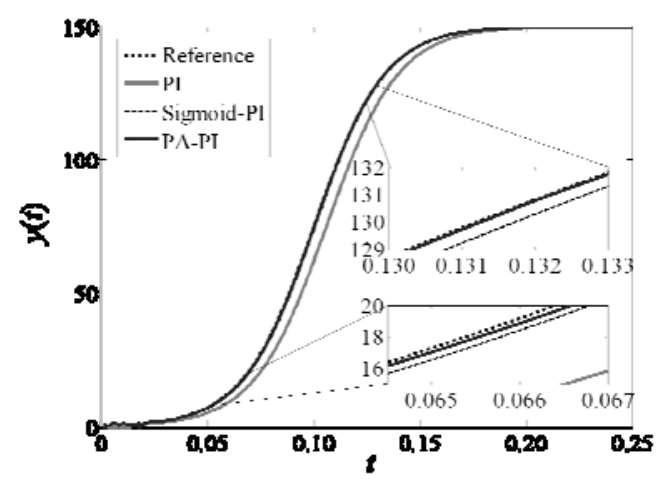

Figure 5. Responses of the angular velocity $y(t)$

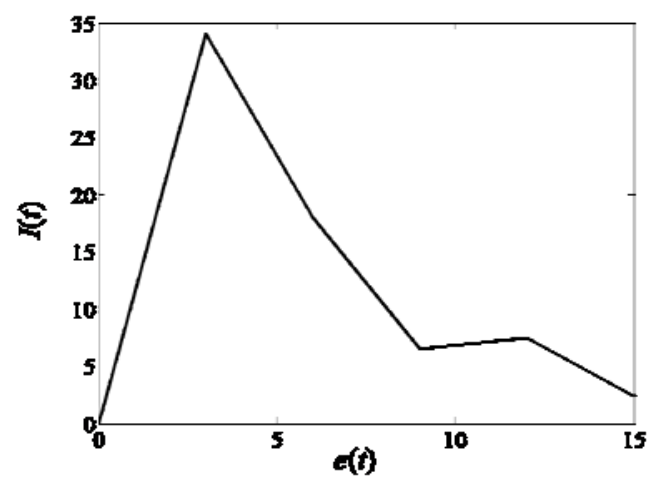

Figure 4. The resulting Piecewise Affine function for the gain $I(t)$

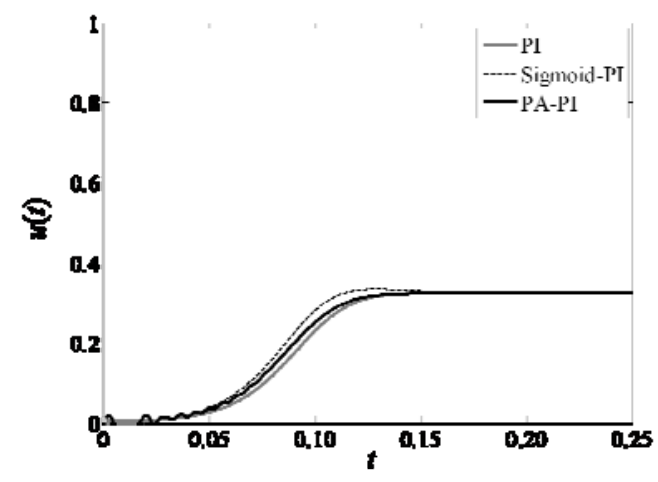

Figure 6. Responses of the angular velocity $u(t)$

Table 3. Performance comparison between PI [7], sigmoid based PI [19] and PA-PI controllers 


\begin{tabular}{cccc}
\hline Controller & PI [7] & Sigmoid- PI[19] & PA-PI \\
\hline $\int_{0}^{0.25} e(t)^{2} d t$ & 6.5190 & 0.0278 & 0.0130 \\
$\int_{0}^{0.25} u(t)^{2} d t$ & 0.0156 & 0.0162 & 0.0161
\end{tabular}

\section{CONCLUSION}

This paper presents a Piecewise Affine Proportional-Integral (PA-PI) controller based SED for buck converter generated DC motor. It justifies that the Piecewise Affine Proportional-Integral (PA-PI) controller has improved the control accuracy, in terms of the responses of the angular velocity and duty ratio input, and the integral square of error and input. In particular, the PA-PI controller based SED is able to closely track the desired angular velocity with small duty ratio input. This fact is also supported by the analysis of the integral square of error and input, where it produces slightly smaller values than the conventional PI and the sigmoid based PI controllers. Thence, this finding justifies the effectiveness of the PA-PI controller in offering more generic class of variable structure PID, resulting in more prospects of controller improvement.

\section{ACKNOWLEDGEMENTS}

The author would like to express his thanks to the Research and Development Department under the grant of RDU1803137.

\section{REFERENCES}

[1] F. Antritter, P. Maurer, and J. Reger, "Flatness based control of a buck converter driven DC motor," in Proc. 4th IFAC Symp. Mechatronic Systems, Heidelberg, Germany, pp. 36-41, 2006.

[2] J. Linares-Flores and H. Sira-Ramirez, "DC motor velocity control through a DC-to-DC power converter," in Proc. IEEE 43rd Conf. Decision Control, Atlantis, The Bahamas, vol. 5, pp. 5297-5302, 2004.

[3] J. Linares-Flores, "Control suave de velocidad de motores de CD mediante convertidores de potencia CD/CD," Ph.D. dissertation, Secci'on de Mecatr'onica del Departamento de Ingenieria El'ectrica del CINVESTAVIPN, Mexico City, Mexico, 2006.

[4] J. Linares-Flores and H. Sira-Ramirez, "A smooth starter for a DC machine: A flatness based approach," in Proc.

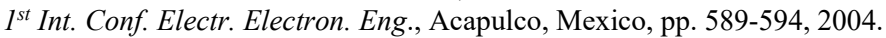

[5] J. Linares-Flores and H. Sira-Ramirez, "Sliding mode-delta modulation GPI control of a DC motor through a buck converter," in Proc. $2^{\text {nd }}$ IFAC Symp. Syst., Struct. Control, Oaxaca, Mexico, pp. 405-409, 2004.

[6] H. El Fadil and F. Giri, "Accounting of DC-DC power converter dynamics in DC motor velocity adaptive control," in Proc. IEEE Int. Conf. Control Appl., Munich, Germany, pp. 3157-3162, 2006.

[7] R.M.T. Raja Ismail, M.A. Ahmad, and M.S. Ramli, "Speed control of buck-converter driven DC motor using LQR and PI: A comparative assessment," in Proc. International Conference on Information Management and Engineering, pp. 651-655, 2009.

[8] R. Silva-Ortigoza, J.R. Garcia-Sanchez, J.M. Alba-Martinez, V.M. Hernandez-Guzm'an, M. Marcelino-Aranda, H. Taud, and R. Bautista-Quintero, "Two-stage control design of a Buck converter/DC motor system without velocity measurements via a $\Sigma$ - $\Delta$-modulator," Math. Probl. Eng., vol. 2013, pp. 1-11, 2013.

[9] M.Z.M. Tumari, M.S. Saealal, M.R. Ghazali, and Y.A. Wahab, "H-infinity with pole placement constraint in LMI region for a buck-converter driven DC motor," in Proc. IEEE Int. Conf. Power Energy, Kota Kinabalu Sabah, Malaysia, pp. 530-535, 2012.

[10] H. Sira-Ramirez and M.A. Oliver-Salazar, "On the robust control of buck-converter DC-motor combinations," IEEE Trans. Power Electron., vol. 28, no. 8, pp. 3912-3922, 2013.

[11] O. Bingol and S. Pac, aci, "A virtual laboratory for neural network controlled DC motors based on a DC-DC buck converter,” Int. J. Eng. Educ., vol. 28, no. 3, pp. 713-723, 2012.

[12] M.A. Ahmad, R.M.T. Raja Ismail, and M.S. Ramli, "Control strategy of buck converter driven DC motor: A comparative assessment," Australian J. Basic Appl. Sci., vol. 4, no. 10, pp. 4893-4903, 2010.

[13] M.A. Ahmad, S. Azuma, and T. Sugie, "Performance analysis of model-free PID tuning of MIMO systems based on simultaneous perturbation stochastic approximation," Expert Systems with Applications, vol. 41, no. 14, pp. 6361-6370, 2014.

[14] I. Boiko, "Variable-structure PID controller for level process," Control Engineering Practice, vol. 21, no. 5, pp.700-707, 2013.

[15] A. Ates, B.B. Alagoz, C. Yeroglu, and H. Alisoy, "Sigmoid based PID controller implementation for rotor control," European Control Conference (ECC), pp. 458-463, 2015.

[16] M.R. Ghazali, M.A. Ahmad, M.F. Mat Jusof, R.M.T. Raja Ismail, "A data-driven neuroendocrine-PID controller for underactuated system based on safe experimentation dynamics," in Proc. of 14th IEEE Colloquium on Signal Processing and Its Applications, pp. 61-66, 2018. 
[17] M.A. Shamseldin, M. Sallam, A.M. Bassiuny, and A.A. Ghany, "A novel self-tuning fractional order PID control based on optimal model reference adaptive system," Int J Power Electronics \& Drive System, vol. 10, no. 1, pp. $230-241,2019$.

[18] P.V. Ramkumar, M.S. Kalavathi, "Fractional Order PID Controlled Interleaved Boost converter Fed Shunt Active Filter System," International Journal of Power Electronics and Drive System, vol. 9, no. 1, pp.126-138, 2018.

[19] M.A. Ahmad, R.M.T. Raja Ismail, "A data-driven sigmoid-based PI controller for buck-converter powered DC motor," in Proc. of IEEE Symposium on Computer Applications and Industrial Electronics, pp. 81-86, 2017.

[20] J.R. Marden, H. Young, G. Arslan, and J. Shamma, "Payoff based dynamics for multi-player weakly acyclic games," in Proc. of $46^{\text {th }}$ IEEE Conference on Decision and Control, pp. 3422-3427, 2007.

[21] J.R. Marden, S.D. Ruben, and L.Y. Pao, "A model-free approach to wind farm control using game theoretic methods," IEEE Transactions on Control Systems Technology, vol. 21, no. 4, pp.1207-1214, 2013.

[22] M.F.F. Kamerul Bieza, N.S. Abdul Shukor, M.A. Ahmad, M.H. Suid, M.R. Ghazali, M.F. Mat Jusof, "A Simplify Fuzzy Logic Controller Based Safe Experimentation Dynamics for Pantograph-Catenary System," Indonesian Journal of Electrical Engineering and Computer Science, Vol. 14, no. 2, 2019.

[23] N.S. Abdul Shukor, M.A. Ahmad, "Data-driven PID controller tuning based on safe experimentation dynamics for control of double-pendulum-type overhead crane," Lecture Notes in Mechanical Engineering, pp. 295-304, 2018.

[24] M.R. Ghazali, M.A. Ahmad, R.M.T. Raja Ismail, "Data-Driven PID control for DC/DC buck-boost converterinverter-DC Motor based on safe experimentation dynamics", in Proc. of IEEE International Conference on Systems, Process and Control, 2018, Accepted.

[25] M.R. Ghazali, M.A. Ahmad, R.M.T. Raja Ismail, "Data-driven Neuroendocrine-PID Tuning Based on Safe Experimentation Dynamics for Control of TITO Coupled Tank System with Stochastic Input Delay", Lecture Notes in Mechanical Engineering, 2019.

\section{BIOGRAPHIES OF AUTHORS}
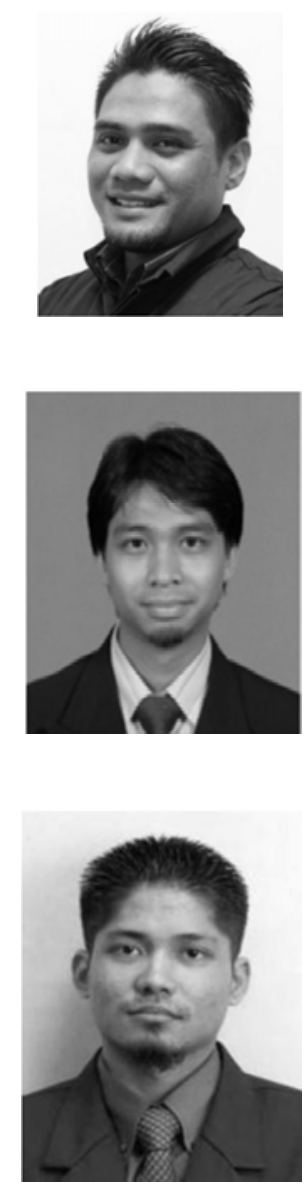

Ir. Mohd Ikhmil Fadzrizan Mohd Hanif is a Professional Engineer with the Board of Engineers Malaysia (BEM) and a Member of Institution of Engineers Malaysia (IEM). He is currently an Instrument Engineer of Operational, Integrity and Engineering Department at Petrofac Malaysia. Ir. Ikhmil holds Bachelor of Electrical Engineering (Control, Instrumentation and Automation) from Universiti Teknikal Malaysia, Melaka (UTeM) in 2007. He has more than 10 years of working experience in Instrumentation and Control engineering in oil, gas and petrochemical industry and also almost 2 years of working experiences as Senior Lecturer in higher education institution major in Electrical and Electronics (E\&E) engineering. His current research interest is data driven piece wise affine PID controller based on Safe Experimentation Dynamics algorithm.

Mohd Helmi Suid received the B.Eng (Hons.) in Electrical and Electronics Engineering from Universiti Teknikal Melaka in 2007. In 2014, he receives M.Sc from School of Electronic and Electrical Engineering, University Sains Malaysia, Penang. He is currently, a lecturer in Faculty of Electrical and Electronics Engineering, University Malaysia Pahang (UMP). His current research interests are digital image processing, vision for robotics application and computational intelligence.

Mohd Ashraf Ahmad received his first degree in B.Eng Electrical Mechatronics and Master Degree in M.Eng Mechatronics and Automatic Control from University of Technology Malaysia (UTM) in 2006 and 2008, respectively. In 2015, he received a Ph.D in Informatics (Systems Science) from Kyoto University. Currently, he is a lecturer in Faculty of Electrical and Electronics Engineering, University Malaysia Pahang (UMP). His current research interests are model-free control, control of mechatronic systems, nonlinear system identification and vibration control. 